\title{
Multi-look polarimetric SAR image filtering using simulated annealing
}

\section{Schou, Jesper}

Published in:

International Geoscience and Remote Sensing Symposium, 2000. Proceedings. IGARSS 2000.

Link to article, DOI:

10.1109/IGARSS.2000.858105

Publication date:

2000

Document Version

Publisher's PDF, also known as Version of record

Link back to DTU Orbit

Citation (APA):

Schou, J. (2000). Multi-look polarimetric SAR image filtering using simulated annealing. In International Geoscience and Remote Sensing Symposium, 2000. Proceedings. IGARSS 2000. (Vol. 3). IEEE. https://doi.org/10.1109/IGARSS.2000.858105

\section{General rights}

Copyright and moral rights for the publications made accessible in the public portal are retained by the authors and/or other copyright owners and it is a condition of accessing publications that users recognise and abide by the legal requirements associated with these rights.

- Users may download and print one copy of any publication from the public portal for the purpose of private study or research.

- You may not further distribute the material or use it for any profit-making activity or commercial gain

- You may freely distribute the URL identifying the publication in the public portal 


\title{
Multi-look Polarimetric SAR Image Filtering using Simulated Annealing
}

\author{
J. Schou, W. Dierking, and H. Skriver \\ Department of Electromagnetic Systems, Technical University of Denmark, DK-2800 Lyngby, Denmark \\ Facsimile: +45 45931634, e-mail: js@emi.dtu.dk, wd@emi.dtu.dk, hs@emi.dltu.dk
}

\begin{abstract}
Based on a previously published algorithm capable of estimating the radar cross-section in synthethic aperture radar (SAR) intensity images, a new filter is presented utilizing multilook polarimetric SAR images. The underlying mean covariance matrix is estimated from the observed sample covariance matrices, and by applying a set of small orientation-dependent filters in an iterative scheme, the input image becomes highly filtered while maintaining most of the structures in the scene. Results using multi-look polarimetric C-band data from the Danish airborne polarimetric SAR, EMISAR, are presented.
\end{abstract}

\section{INTRODUCTION}

The elements of the scattering matrix are measured for each resolution cell by a polarimetric SAR system. The covariance matrix (CM) is formed from the scattering matrix, and often standard multi-look processing involving averaging of neighboring CMs is applied to reduce the effect of the speckle inherent in SAR images. As the degree of multi-looking is limited by the need to preserve structures in the scene, additional filtering is often applied using one of several available polarimetric SAR filters.

In this work a new algorithm capable of filtering polarimetric images is presented. It is based on the work by White [1], McConnell et al [2], and Oliver and Quegan [3], but rather than estimating the radar cross-section (RCS) given the observed intensity image, the algorithm estimates the mean CM based on the observed polarimetric images, thus providing better estimates of all the polarimetric parameters characterizing the surface.

The algorithm is part of the class of image restoration algorithms, where the underlying image is restored from the observed image. Modeling the image as a Markov random field (MRF), the image restoration is expressed as an estimation problem. As a MRF follows the Gibbs distribution, this estimation problem is equivalent to an energy minimization problem, which is solved using simulated annealing (SA) [4][5]. The SA algorithm is iterative by nature, hence we can employ small filters in the restoration. Also, by choosing between a set of orientation-dependent filters, the resulting mean CM estimate provided by the algorithm is highly speckle reduced for the distributed targets, while most of the structures in the images are preserved.

\section{MARKOV RANDOM FIELDS AND SIMULATED ANNEALING}

If $\mathcal{S}=\left\{s_{1}, s_{2}, \ldots, s_{N}\right\}$ denotes the $N$ pixels of a 2-D image, and the pixel values $\mathbf{x}=\left\{x_{1}, \ldots, x_{N}\right\}$ are realizations of the stochastic variables $\mathbf{X}=\left\{X_{1}, \ldots, X_{N}\right\}$ defined on $\mathcal{S}$, then $\mathbf{X}$ is a random field [5].

For the Markov random field defined on $\mathcal{S}$, the probability of a particular configuration (image) of $\mathbf{X}$ follows a Gibbs distribution [4][5]

$$
P(\mathrm{X}=\mathbf{x})=\frac{1}{Z} \mathrm{e}^{-\frac{u(\mathbf{x})}{T}}, \mathbf{x} \in \Omega
$$

where $T$ is called the temperature, and $Z$ is a normalization function called the partition function. The energy function, $U(\mathbf{x})$, depends on the pixel values contained in the local neighborhood.

When information on a process underlying an observed process is needed, Bayesian methods are often applied. If the underlying process is called $\mathbf{X}$, and the observed process $\mathbf{Y}$, then Bayes rule states that the $a$ posteriori conditional probability $P(\mathbf{x} \mid \mathbf{y})$ can be found from the likelihood function $P(\mathbf{y} \mid \mathbf{x})$ and the a priori probability $P(\mathbf{x})$ by

$$
P(\mathbf{x} \mid \mathbf{y}) \propto P(\mathbf{y} \mid \mathbf{x}) P(\mathbf{x})
$$

When $\mathbf{X}$ is modeled as a MRF, the $a$ posteriori probability can be expressed as

$$
P(\mathbf{x} \mid \mathbf{y})=\frac{1}{Z} \mathrm{e}^{-\frac{U(x \mid y)}{T}}
$$

using the a posteriori conditional energy function $U(\mathbf{x} \mid \mathbf{y})$. If knowledge about the a priori probability is omitted, the estimate, $\hat{\mathbf{x}}$, maximizing ( 3 ) is the maximum likelihood (ML) estimate, and for the mean CM restoration algorithm the aim is to estimate the underlying, unspeckled image $\mathbf{X}$ from the observed, speckled image $\mathbf{Y}$ using the ML estimate.

The global maximum of the a posteriori distribution in (3) is equivalent to the global minimum of the a posteriori conditional energy function. This latter minimum is found using the stochastic optimization method simulated annealing. SA searches for the configuration $\mathbf{x}$ that minimizes $U(\mathbf{x})$. It is iterative by nature, where a new configuration $\mathrm{x}^{j}$ for iteration $j$ is found from the previous configuration $x^{j-1}$ by applying a generation mechanism and accepting the new configuration using an acceptance criterion based on the energy divergence $\Delta U=U\left(\mathbf{x}^{j}\right)-U\left(\mathbf{x}^{j-1}\right)$, and the temperature. At the end 


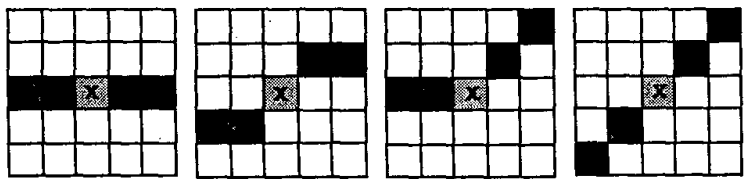

Fig. 1: 4 connection filters from the set of 16 filters used by the generation mechanism to find the new estimate of $\mathbf{C}$; the remaining 12 filters are rotated and mirrored versions of these 4 . The $x$ marks the central pixel and the dark pixels are the neighbors.

of the minimization the global minimum configuration, $\hat{\mathbf{x}}$, is ideally reached [4][5].

The generation mechanism derives the new pixel value at a given pixel from a set of $N_{c}$ connection filters [1]. In this work we apply 16 filters, 4 of which are seen in Fig. 1; each filter has $n_{n}$ neighbors and we use $n_{n}=4$. For each filter the new value of the central pixel is based on the original value of the central pixel and the present values of the neighboring pixels.

\section{COVARIANCE MATRIX RESTORATION}

In the following we will assume that reciprocity holds [3], thus the Hermitian sample CM $\mathbf{\Sigma}$ at each pixel can be expressed as

$$
\boldsymbol{\Sigma}=4 \pi\left\langle\left[\begin{array}{ccc}
\left|S_{h b}\right|^{2} & S_{h h} S_{h v}^{*} & S_{h h} S_{v v}^{*} \\
S_{h v} S_{h h}^{*} & \left|S_{h v}\right|^{2} & S_{h v} S_{v v}^{*} \\
S_{v v} S_{h h}^{*} & S_{v v} S_{h v}^{*} & \left|S_{v v}\right|^{2}
\end{array}\right]\right\rangle
$$

where $*$ denotes complex conjugation. The sample covariance matrix follows a complex Wishart distribution, which is a function of the number of looks $L$ and the Hermitian mean CM C [6]

$$
p(\boldsymbol{\Sigma} \mid \mathbf{C})=\frac{L^{L p}|\boldsymbol{\Sigma}|^{L-p} \mathrm{e}^{-L \mathrm{tr}\left(\mathbf{C}^{-1} \mathbf{\Sigma}\right)}}{\pi^{\frac{1}{2} p(p-1)} \prod_{j=1}^{p} \Gamma(L-j+1)|\mathbf{C}|^{L}}
$$

where $p$ is the dimension of $\boldsymbol{\Sigma}$, ie. $p=3$ assuming reciprocity, $|\ldots|$ and $\operatorname{tr}(. .$.$) denote the determinant and trace, re-$ spectively, and $\Gamma(\ldots)$ is the Gamma function. Goodman [6] shows, that $\boldsymbol{\Sigma}$ is an unbiased estimate of the mean $\mathrm{CM}, \mathrm{C}$, and as the mean $\mathrm{CM}$ is the quantity to be restored by the algorithm, (5) represents the likelihood function.

Using the connection filters the new estimate of the mean $\mathrm{CM}$ for the central pixel is based on the original sample $\mathrm{CM}$ at the central pixel of the connection filter, and the present estimates of the mean $\mathrm{CM}$ for the neighbors. Denoting the original, central sample $C M$ as $\boldsymbol{\Sigma}_{0}$, and the neighboring mean $\mathrm{CM}$ estimates as $\mathbf{Z}_{1}, \ldots, \mathbf{Z}_{n_{n}}$ we find the a posteriori probability as

$$
P\left(\mathbf{C}_{i k} \mid \mathbf{\Sigma}_{0}, \mathbf{Z}_{1 k}, \ldots, \mathbf{Z}_{n_{n} k}\right) \propto P\left(\boldsymbol{\Sigma}_{0}, \mathbf{Z}_{1 k}, \ldots, \mathbf{Z}_{n_{n} k} \mid \mathbf{C}_{i k}\right)
$$

where suffix $i$ denotes the pixel and suffix $k$ denotes the connection filter. The a priori probability, $P\left(\mathbf{C}_{i k}\right)$, is omitted, as only the ML estimate is derived. The CMs entering (6) follow a complex Wishart distribution having the same mean CM. The original sample $\mathrm{CM}$ has $L$ number of looks, and the neighboring CMs have the effective number of looks $\alpha$, which is estimated in a window around the central pixel.

For each of the connections, the log likelihood function at site $i$ is given as $L f\left(\mathbf{C}_{i k}\right)=\log P\left(\boldsymbol{\Sigma}_{0}, \mathbf{Z}_{1 k}, \ldots, \mathbf{Z}_{n_{n} k} \mid \mathbf{C}_{i k}\right)$. The ML estimate of the mean CM is derived by setting $\frac{d L f\left(C_{i k}\right)}{d C_{i k}}$ $=0$, with 0 being the $3 \times 3$ null matrix, resulting in

$$
\hat{\mathbf{C}}_{i k}=\frac{\boldsymbol{\Sigma}_{0}+\frac{\alpha}{L}\left(\mathbf{Z}_{1 k}+\ldots+\mathbf{Z}_{n_{n} k}\right)}{1+n_{n} \frac{\alpha}{L}}
$$

It is seen, that the locally estimated number of looks, $\alpha$, controls the degree of averaging. For a smooth surface $\alpha$ is high, resulting in $\hat{\mathbf{C}}_{i k} \approx \frac{1}{n_{n}}\left(\mathbf{Z}_{1 k}+\ldots+\mathbf{Z}_{n_{n} k}\right)$, whereas a discontinuity in the local window makes $\alpha$ small, and thus gives a higher priority to the original sample CM. This expression is a generalization of the RCS estimate [1][2][3] given as

$$
\hat{\sigma}_{i k}=\frac{I_{0}+\frac{\alpha}{L}\left(x_{1 k}+x_{2 k}\right)}{1+2 \frac{\alpha}{L}}
$$

with $\hat{\sigma}_{i k}$ being the estimated RCS, $I_{0}$ the original sample intensity and $x_{1}$ and $x_{2}$ the current estimiates of the RCS at the 2 neighboring pixels.

The mean $\mathrm{CM}$ restoration is performed as an energy minimization process, with the a posteriori energy function given as

$$
\begin{aligned}
& U_{M L}\left(\mathbf{C}_{i k} \mid \mathbf{\Sigma}_{0}, \mathbf{Z}_{1 k}, \ldots, \mathbf{Z}_{N_{n} k}\right)= \\
& -\ln \left(P\left(\boldsymbol{\Sigma}_{0}, \mathbf{Z}_{1 k}, \ldots, \mathbf{Z}_{N_{n} k} \mid \mathbf{C}_{i k}\right)\right)
\end{aligned}
$$

It is seen, that a high likelihood probability results in a small a posteriori energy function, as needed when the restoration is performed as an energy minimization process. For each of the connections, the probability

$$
P\left(\mathbf{C}_{i k} \mid \Sigma_{0}, \mathbf{Z}_{1 k}, \ldots, \mathbf{Z}_{n_{n} k}\right)=\frac{1}{Z} \mathrm{e}^{-\frac{v_{M L}\left(\mathbf{C}_{i k} \mid \Sigma_{0}, \mathbf{z}_{1 k}, \ldots, \mathbf{z}_{n_{n k}}\right)}{T}}
$$

is calculated with the partition function given as

$$
Z=\sum_{k} \mathrm{e}^{-\frac{U_{M L}\left(\mathbf{C}_{i k} \mid \Sigma_{0}, \mathbf{z}_{1 k} \ldots, \mathbf{z}_{n_{n} k}\right)}{T}}
$$

The connection $k_{0}$ resulting in the highest a posteriori probability (the lowest a posteriori energy) is identified, and subsequently accepted or rejected based on the Metropolis criterion $[4][5]$.

\section{EXPERIMENTAL RESULTS}

The CM restoration algorithm is tested on fully polarimetric 13-look C-band data from the Danish airborne SAR, EMISAR 


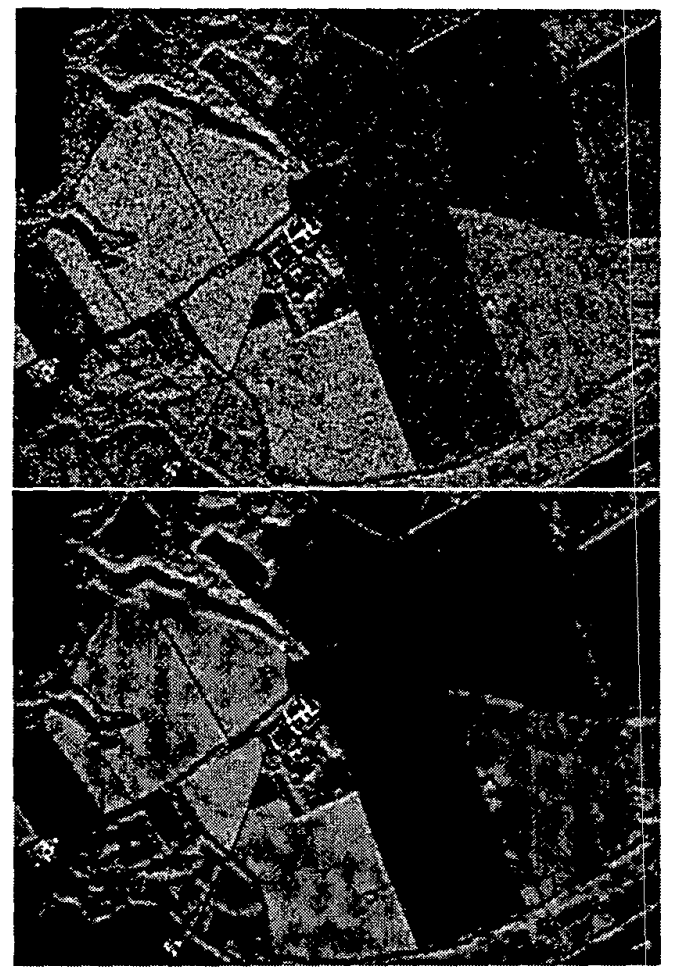

Fig. 2: ML Covariance matrix estimation using 100 iterations, and a local $5 \times 5$ window for estimating $\alpha$. Top: $\sigma_{h h}$ original, Bottom: $\hat{\sigma}_{h h}$

[7]. The test site, shown at the top in Fig. 2, includes a number of natural and man-made targets, and these 13-look data having a $5 \times 5 \mathrm{~m}$ ground pixel spacing are in the following denoted as the original data. At the bottom of Fig. $2, \hat{\sigma}_{h . h}$ from the mean $\mathrm{CM}$ restoration is shown. The homogeneous areas in the restored image appear very smooth, while most of the structures are maintained in the image. Estimating the effective number of looks for the 4 large fields in the right part of Fig. 2 yields $271,448,539$, and 255 when estimated from $\hat{\sigma}_{h h}$, compared to $9.8,8.4,10.5$, and 9.6 when estimated from $\sigma_{h h}$. The estimate $\hat{\sigma}_{h h}$ for the entire scene is, however, biased by $-0.88 \mathrm{~dB}$. Fig. 3 shows the original and restored coherence, $\rho_{h h, v v}=\frac{\left\langle S_{h h} S_{v v}^{*}\right\rangle}{\sqrt{\left|S_{h h}\right|^{2}\left|S_{v v}\right|^{2}}}$, and again the homogeneous areas in the restored image appear highly filtered.

\section{CONCLUSION}

A new approach for mean CM estimation is presented, working on polarimetric SAR data and capable of achieving a very high degree of speckle reduction. The restored mean $\mathrm{CM}, \hat{\mathbf{C}}$, contains the full polarimetric information, although a bias in the estimate is observed, and $\hat{\mathbf{C}}$ can be used by any application using polarimetric data, e.g. target detection, segmentation, and classification.

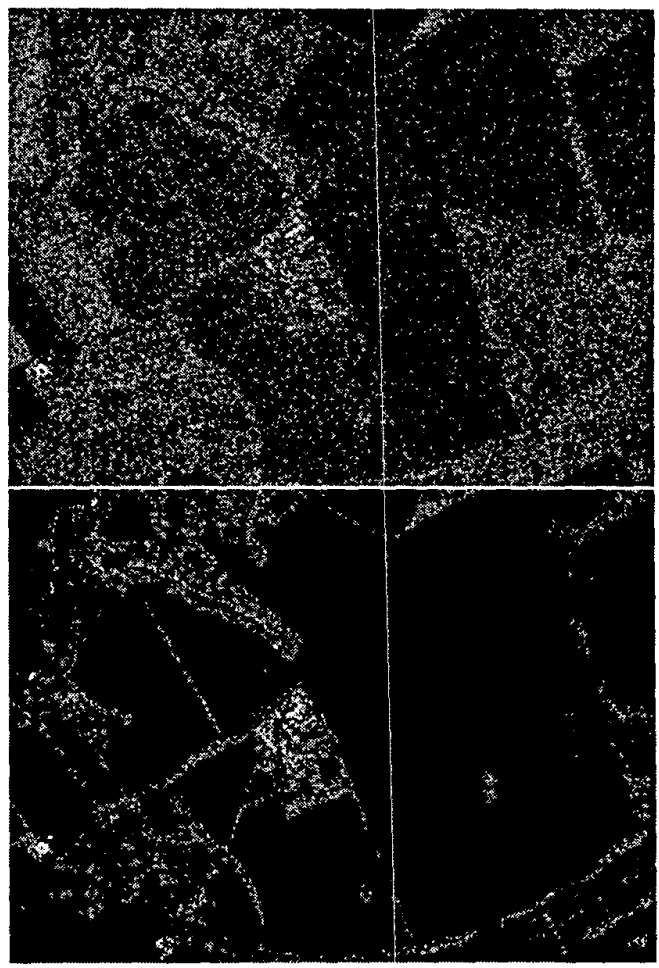

Fig. 3: ML Covariance matrix estimation using 100 iterations, and a local $5 \times 5$ window for estimating $\alpha$. Top̣: $\left|\rho_{h h, v v}\right|$ original, Bottom: $\left|\hat{\rho}_{h h, v v}\right|$

\section{REFERENCES}

[1] R.G. White "A simulated annealing algorithm for SAR and MTI image cross-section estimation", SPIE, Vol. 2316, 1994 pp. 137-145

[2] I. McConnell, R. White, C. Oliver and R. Cook "Radar Cross-Section Estimation of SAR Images", SPIE, Vol. 2584,1995 pp. 164-175

[3] C. Oliver and S. Quegan "Understanding Synthetic Aperture Radar Images", Artech House, 1998

[4] D. Geman "Random Fields and Inverse Problems in Imaging", Springer-Verlag, Lecture Notes in Mathematics, Vol. $1427,1990 \mathrm{pp}$.

[5] S.Z. Li "Markov Random Field Modeling in Computer Vision", Springer-Verlag, Computer Science Workbench, $1995 \mathrm{pp}$

[6] N.R. Goodman "Statistical Analysis Based on a Certain Multivariate Complex Gaussian Distribution (an Introduction)" , Ann. Math. Stat., Vol. 34, 1963 pp. 152-177

[7] E.L. Christensen, N. Skou, J. Dall, K.W. Woelders, J.H. Jørgensen, J. Granholm and S.N. Madsen "EMISAR: An Absolutely Calibrated Polarimetric L- and C-band SAR" , IEEE Transactions on Geoscience and Remote Sensing, Vol. 36, No. 6, November, 1998 pp. 1852-1865 